\title{
Incorporating Distance Domination in Multiobjective Evolutionary Algorithm
}

\author{
Praveen K. Tripathi, Sanghamitra Bandyopadhyay, and Sankar K. Pal \\ Machine Intelligence Unit, \\ Indian Statistical Institute, \\ 203 B. T. Road, Kolkata 700108 \\ \{praveen_r, sanghami, sankar\}@isical.ac.in
}

\begin{abstract}
In this article we propose a novel distance domination parameter and describe a multiobjective evolutionary concept called distance domination based multiobjective evolutionary algorithm (DBMEA). The distance parameter drives the algorithm faster in approximating the Pareto optimal front. To ensure proper diversity in the solutions of the non-dominating set, a new method for incorporating diversity is explained. The DBMEA has been compared with the NSGA-II algorithm on different test functions using different performance measures.
\end{abstract}

\section{Introduction}

In this article, a new evolutionary multiobjective optimization (MOO) algorithm called "Distance-domination Based Multiobjective Evolutionary Algorithm (DBMEA)" is proposed. DBMEA uses the concept of distance domintaion parameter which refines the fitness function and helps in better convergence to the true Pareto-front. It incorporates non-dominated sorting and crowded-tournament selection as in NSGA-II [1]. The diversity parameter used here is also novel. DBMEA incorporates the concept of elitism, using archive concept as in SPEA2 [2. Comparative results of DBMEA and NSGA-II are provided for different standard test functions in terms of four performance measures.

\section{Basic Principles}

A general minimization problem of $M$ objectives can be mathematically stated as:

- Minimize : $f(x)=\left[f_{i}(x), i=1, \ldots, M\right]$, subject to : $g_{j}(x) \leq 0, \quad j=1,2, \ldots, J$, and

$h_{k}(x)=0, \quad k=1,2, \ldots, K$, where $f_{i}(x)$ is the $i^{t h}$ objective function, $g_{j}(x)$ is the $j^{\text {th }}$ inequality constraint, and $h_{k}(x)$ is the $k^{\text {th }}$ equality constraint.

The MOO problem then reduces to finding $x$ such that $f(x)$ is optimized. A solution is said to dominate another solution if it is not worse than that solution in all the objectives and is strictly better than that in at least one objective. The solutions over the entire solution space that are not dominated by any other solution are called Pareto-optimal solutions. 


\section{Distance-Domination Based Multiobjective Evolutionary Algorithm (DBMEA)}

The basic structure of DBMEA is given in Algorithm 1. Different steps are described below in brief.

As is normally done, the parameters of the search space in DBMEA are encoded as string like structures called chromosomes. A fitness value is assigned to each chromosome that denotes the degree of goodness of the encoded solution. The fitness $F_{i}$ of the $i^{\text {th }}$ chromosome is computed as:

$$
F_{i}=f_{r i}+f_{d i}
$$

where $f_{r i}$ is the rank parameter as defined in [1] and $f_{d i}$ is the distance domination parameter proposed in this article.

- Rank Parameter $\left(f_{r i}\right)$ : The parameter $f_{r i}$ is a measure of a solution's nondominance. For computing $f_{r i}$, non-dominated sorting [1] is used to first determine the non-dominated fronts. $f_{r i}$ is then set equal to the front number where $i^{\text {th }}$ solution appears 1 .

- Distance Domination Parameter $\left(f_{d i}\right)$ : This new parameter is calculated as the normalized Euclidean distance of a dominated solution from its nearest nondominated solution on the first front. The significance of this parameter is to give higher priority to those dominated solutions, that are closer to their dominating solution on the first front. As an example considering two functions minimization in Figure 1(a), the solutions ' $g$ ', ' $h$ ' and ' $j$ ' are dominated by solution ' $c$ ' on the first front. Normally, ' $g$ ', ' $h$ ' and ' $j$ ' would have the same status. However, it can be observed that ' $h$ ' is much closer to the first front. It is our intuition that ' $h$ ' should get higher priority over ' $g$ ' and ' $j$ '. The parameter $f_{d i}$ ( $=$ ' $d 1$ ' for, ' $g$ ', ' $d 2$ ' for ' $h$ ' and ' $d 3$ ' for ' $j$ ') takes care of this fact.

The elitism concept in DBMEA is similar to that in SPEA-2 2]. An archive of fixed size (equal to the size of the population, $N$ ) is maintained, that gets updated at each iteration. After computation of the fitness archive is first filled by non-dominated solutions of the current archive and population, followed by the best (in terms of $F_{i} \mathrm{~s}$ ) remaining solutions till the archive is full. In case of archive overflow at any stage, the density paramater $d_{i}$ is used to truncate the archive. The parameter $\left(d_{i}\right)$ (which is similar to crowded-distance [1], with some modification) is computed as the distance of each solution to its immediate next neighbour summed over each of the $M$ objectives. For example in Figure 1(b), ' $d 1$ ' and ' $d 2$ ' correspond to the distances along $f 1$ for solutions ' $e$ ' and ' $f$ ' respectively. Similarly ' $d 4$ ' and ' $d 3$ ' correspond to the distance along $f 2$ for solutions ' $c$ ' and ' $b$ ' respectively.

After the fitness assignment, DBMEA uses density-based binary tournament selection on the archive to create the mating pool. This concept is inspired by the crowded-tournament selection [1, where crowded-distance has been used in order to overcome the tie in fitness values. Here if we get the solutions with the same $F_{i}$ values, then we conside $d_{i}$ and prefer the less crowded solution. The DBMEA uses single-point crossover and bit-wise mutation operation. 
Algorithm 1. $O_{f}=\operatorname{DBMEA}\left(P_{t}, A_{t}, N, C\right) /{ }^{*} P_{t}$ : population at iteration t, $A_{t}$ : archive at iteration $\mathrm{t}, \mathrm{N}$ : size of the population and archive, $\mathrm{C}$ : maximum number of iterations. $O_{f}$ : the final output */

1. $t=0$, randomly initialize $P_{0}$ and compute the objectives, $A_{0}=\Phi$.

2. non-dominated sorting on $P_{t} \cup A_{t}$.

3. $\forall i, \quad i \in\left\{P_{t} \cup A_{t}\right\}$, calculate $f_{r i}, f_{d i}$ and $d_{i}$.

$F_{i}=f_{r i}+f_{d i}$

4. $A_{t+1} \leftarrow$ non-dominated solutions from $P_{t} \cup A_{t}$

- if $\left\{\left|A_{t+1}\right|\right\}>N$, truncate $A_{t+1}$ to size $N$ using $d_{i}$

- if $\left\{\left|A_{t+1}\right|\right\}<N$, copy $\left\{N-\left|A_{t+1}\right|\right\}$ dominated solutions from $P_{t} \cup A_{t}$ to $A_{t+1}$.

5. If $t \geq C$ or other terminating condition is fulfilled, $O_{f} \leftarrow A_{t+1}$ and stop.

6. Genetic Operations:

- Density-based binary tournament selection on $A_{t+1}$ to get the mating pool.

- Single-point crossover and bitwise mutation performed on mating pool to get $P_{t+1}$, compute objectives of $P_{t+1}$.

$t \leftarrow t+1$; goto $(2)$.

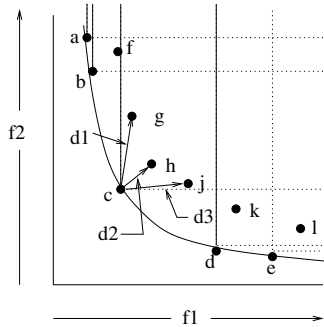

(a)

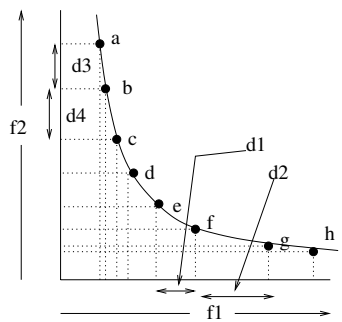

(b)

Fig. 1. (a): $f_{d i}$ parameter (b): diversity parameter

\section{Simulation Study}

DBMEA and NSGA-II have been compared on ten standard test problems. All the test problems studied in the present article involve minimization of the functions. Though the test problems have real domain, the binary encoded implementation of the algorithms (thus discretizing the domain, in practice) have been studied. The parameters used in these experiments are: population size: 100, number of iterations: 250, crossover-probability: 0.9, mutation probability: inversely proportional to the chromosome length, chromosome-length: 10 bits per 
variable. Ten runs of the algorithms were executed on each test problem, the mean and variance of the performance measures over these runs are reported.

\section{Test Problems and Performance Measures}

The set of test problems used in this article includes SCH1 and SCH2 [3], FON [4, KUR [5], POL [6, ZDT1, ZDT2, ZDT3, ZDT4 and ZDT6 [7. The performance of DBMEA and NSGA-II is compared with respect to four performance measures: the convergence measure $\Upsilon$ [1], diversity measure $\Delta[1$, purity value [8] and minimal-spacing 8 . The larger value of the purity measure and the smaller values of $\Upsilon, \Delta$ and minimal spacing parameters signify the better performance. The details of the measures may be found in the respective references.

\section{Results}

Tables 1 and 2 represent the convergence measure and diversity measure, and the purity and minimal spacing measure respectively.

Table 1. Convergence and Diversity measure

\begin{tabular}{|c|c|c|c|c|c|c|c|}
\hline \multicolumn{8}{|c|}{ Convergence Measure $\Upsilon$} \\
\hline & \begin{tabular}{l|l}
$\mathrm{CH} 1$ & $\mathrm{SCH} 2$ \\
\end{tabular} & FON & ZDT1 & ZDT2 & ZDT3 & ZDT4 & ZD \\
\hline \multirow{2}{*}{$\begin{array}{c}\text { NSGA-II(Mean) } \\
\text { (Variance) }\end{array}$} & \begin{tabular}{l|l}
44 & 0.
\end{tabular} & 32 & & 0.0027 & $0.002 \vdots$ & 2.7681 & \\
\hline & 0.0 & & & & & & \\
\hline \multirow{2}{*}{$\begin{array}{c}\text { DBMEA(Mean) } \\
\text { (Variance) }\end{array}$} & \begin{tabular}{l|l}
0.0132 & 0.0167 \\
\end{tabular} & .0012 & 0.00 & 0.0030 & 0.0033 & 1.2338 & 0.00 \\
\hline & \begin{tabular}{|l|l|}
0.0000 & 0.0000 \\
\end{tabular} & 0.0000 & & 0.0000 & 0.0000 & 1.2069 & \\
\hline \multicolumn{8}{|c|}{ Diversity Measure $\Delta$} \\
\hline Algor & \begin{tabular}{l|l} 
SCH1 & SCH2
\end{tabular} & FON & ZDT1 & ZDT2 & ZDT3 & ZDT4 & ZDT \\
\hline \multirow{2}{*}{$\begin{array}{c}\text { NSGA-II(Mean) } \\
\text { (Variance) }\end{array}$} & \begin{tabular}{|l|l|}
0.5763 & 1.4845 \\
\end{tabular} & 0.77 & 0.90 & 0.8322 & 0.7037 & 0.9474 & 0.945 \\
\hline & \begin{tabular}{|l|l|}
0.0068 & 0.0499 \\
\end{tabular} & .0004 & 0.00 & 0.0059 & 0.0025 & 0.0011 & 0.001 \\
\hline \multirow{2}{*}{$\begin{array}{c}\text { DBMEA (Mean) } \\
\text { (Variance) }\end{array}$} & \begin{tabular}{|l|l|}
0.3639 & 1.2623 \\
\end{tabular} & 0.74 & $0.5^{\prime}$ & 0.3903 & 0.7304 & 0.8075 & 0.81 \\
\hline & \begin{tabular}{|l|l|}
0.0291 & 0.07 \\
\end{tabular} & & & 0001 & 0 & & \\
\hline
\end{tabular}

Table 2. Purity and Minimal Spacing measure

\begin{tabular}{|c|c|c|c|c|c|c|c|c|}
\hline & \multicolumn{4}{|c|}{ NSGA-II } & \multicolumn{4}{c|}{ DBMEA } \\
\hline Test Function & \multicolumn{2}{|c|}{ Purity } & Minimal Spacing & \multicolumn{2}{c|}{ Purity } & Minimal Spacing \\
\hline & Mean & Variance & Mean & Variance & Mean & Variance & Mean & Variance \\
\hline SCH1 & 0.672 & 0.0639 & 0.0104 & 0.00 & 0.826 & 0.0528 & 0.0044 & 0.00 \\
\hline SCH2 & 0.808 & 0.0107 & 0.0524 & 0.00 & 0.818 & 0.0213 & 0.0497 & 0.00 \\
\hline KUR & 0.811 & 0.0055 & 0.0897 & 0.00 & 0.943 & 0.0008 & 0.0877 & 0.00 \\
\hline POL & 0.646 & 0.0465 & 0.0705 & 0.00 & 0.756 & 0.0403 & 0.0699 & 0.00 \\
\hline FON & 0.680 & 0.0018 & 0.0084 & 0.00 & 0.944 & 0.0004 & 0.0005 & 0.00 \\
\hline ZDT1 & 0.989 & 0.0003 & 0.0113 & 0.00 & 0.397 & 0.0577 & 0.0069 & 0.00 \\
\hline ZDT2 & 0.998 & 0.0004 & 0.0142 & 0.00 & 0.187 & 0.0116 & 0.0114 & 0.00 \\
\hline ZDT3 & 0.957 & 0.0080 & 0.0358 & 0.00 & 0.410 & 0.0233 & 0.0369 & 0.00 \\
\hline ZDT4 & 0.200 & 0.1778 & 0.0164 & 0.00 & 0.800 & 0.1778 & 0.0064 & 0.00 \\
\hline ZDT6 & 1.000 & 0.0000 & 0.0146 & 0.00 & 1.000 & 0.0000 & 0.0087 & 0.00 \\
\hline
\end{tabular}




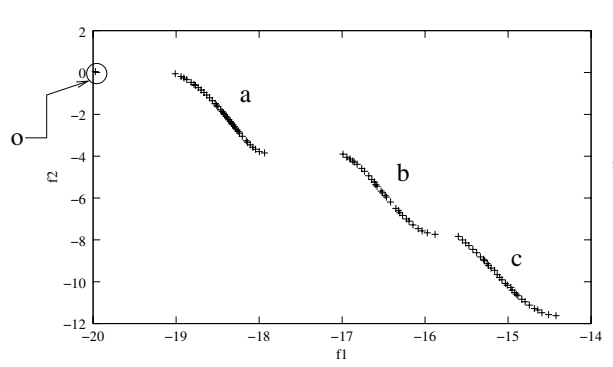

(a)

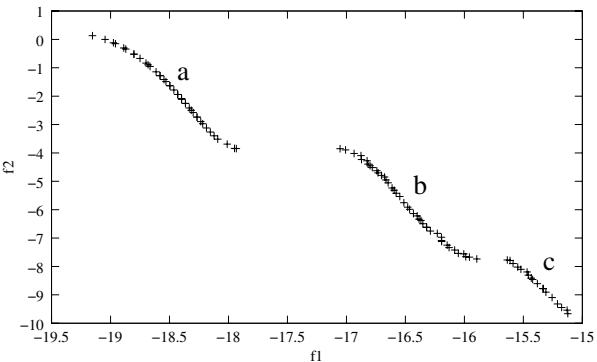

(b)

Fig. 2. Final Pareto-fronts of (a): DBMEA and (b): NSGA-II on KUR

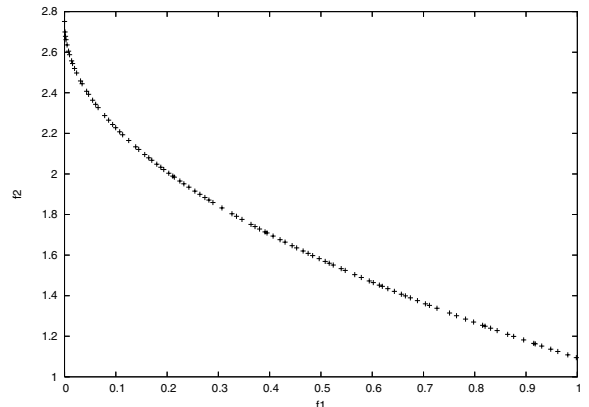

(a)

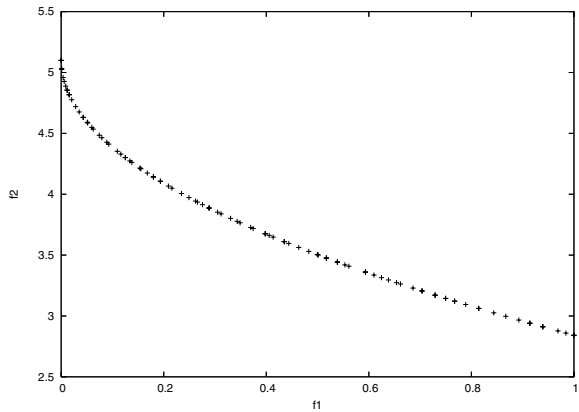

(b)

Fig. 3. Final Pareto-fronts of (a): DBMEA and (b): NSGA-II on ZDT4

As can be seen from Table 1, DBMEA has better convergence on test problems SCH1, SCH2, FON, ZDT4 and ZDT6, whereas NSGA-II has better convergence on functions ZDT1, ZDT2 and ZDT3. However it is interesting to note that except for ZDT3, DBMEA provides a better value of the diversity measure $\Delta$ for all other test problems. Again from Table 2 , it is evident that DBMEA has better purity value for the test functions SCH1, SCH2, KUR, POL, FON, ZDT4 and ZDT6 compared to NSGA-II. The minimal spacing parameter shows that DBMEA has attained better spread of the solutions on the front for all test functions except ZDT3. Figures 2 and 3 show the final non-dominated fronts obtained by DBMEA and NSGA-II for two of the test functions (results for the others were almost similar). The better performance of DBMEA in terms of convergence and diversity is quite evident from Figure 3. In Figure 2, DBMEA is able to obtain the disjoint Pareto-optimal point ' $O$ ' at $(-20,0)$ and the third disconnected component ' $c$ ' of the front, while NSGA-II fails to do that properly. From the results it can be concluded that DBMEA has comparable results with, often better than, NSGA-II in terms of the convergence to the Pareto-optimal front. In terms of the attainment of diversity on the Pareto-optimal front DBMEA is generally better than NSGA-II. 


\section{Discussion and Conclusions}

In the present article, we have introduced a novel concept of measuring fitness function in MOEA by introducing a distance domination factor. A new way of computing density reflecting the diversity is explained that has resulted in much better spread of the solutions on the Pareto-optimal front. The MOEA based on this concept is not only good in approximating Pareto-optimal front, but also performs better in terms of diversity of the solutions on the front. Though the experiments done in this article have considered a good number of test problems with varying characterstics, a theoretical study dealing with the generalised performance of DBMEA is currently under investigation. It may be noted that the notion of distance domination is a general concept that can be used in other MOO algorithms also.

Acknowledgements. The present work has been partially supported by INSA funded project $N o . B S / Y S P / 36 / 886$.

\section{References}

1. Deb, K., Pratap, A., Agarwal, S., Meyarivan, T.: A Fast and Elitist Multi-objective Genetic Algorithm: NSGA-II. Technical Report 200001, Kanpur Genetic Algorithms Laboratory (KanGAL), Indian Institute of Technology Kanpur, India (2000)

2. Zitzler, E., Laumanns, M., Thiele, L.: SPEA2: Improving the Strength Pareto Evolutionary Algorithm. Technical Report TIK-103, Computer Engineering and Network Laboratory (TIK), Swiss Fedral Institute of Technology (ETH), Gloriastrasse 35, CH-8092 Zurich, Swidzerland (2001)

3. Schaffer, J.D.: Some Experiments in Machine Learning using Vector Evaluated Genetic Algorithm. PhD thesis, Vanderbilt University, Nashville,TN (1984)

4. Fonseca, C.M., Fleming, P.J.: An Overview of Evolutionary Algorithms in Multiobjective Optimization. Evolutionary Computation Journal 3 (1995) 1-16

5. Kursawe, F.: A Variant of Evolutionary Strategies for Vector Optimization. In: In Parellel Problem Solving from Nature I (PPSN-I). (1990) 193-197

6. Poloni, C., Giurgevich, A., Onesti, L., Pediroda, V.: Hybridization of a Multiobjective Genetic Algorithm, a Neural Network and a Classical Optimizer for Complex Design Problem in Fluid Dynamics. Computer Methods in Applied Mechanics and Engineering (2000) 403-420

7. Zitzler, E., Deb, K., Thiele, L.: Comparison of Multiobjective Evolutionary Algorithms: Empirical Results. Evolutionary Computation Journal 8 (2000) 125-148

8. Bandyopadhyay, S., Pal, S.K., Aruna, B.: Multi-Objective GAs, Quantitative Indices, and Pattern Classification. IEEE Transaction on Systems, Man, and Cybernetics-Part B: Cybernetics 34 (2004) 2088-2099 